\title{
Robotics in the Construction Industry: Mass Customization or Digital Crafting?
}

\author{
Ingrid Paoletti and Roberto Stefano Naboni \\ Politecnico di Milano, Building Environment Science \& Technology, Milano, Italy \\ ingrid.paoletti@polimi.it, roberto.naboni@gmail.com
}

\begin{abstract}
The paper discusses the advancement in the mass-customization of building components referring to Robot-Assisted Manufacturing. It is presented how the contemporary employment of Robotics offers a perspective of flexible alternative to traditional serial production system. Different Robot-Assisted fabrication methods are discussed through built experimental case studies at different scales. It is finally argued how Robotic production in architecture is significantly shifting the approach in design towards a model including material and fabrication constraints.
\end{abstract}

Keywords: Mass-Customization, Digital Fabrication, Parametric Design, Robot-Assisted Manufacturing, Additive Production.

\section{Component Customization in the Construction Industry}

Typically construction methods in building industry are based on the principles of mass-production: standardization, modularization and production lines. Compared to other fields, construction industry has not been characterized by the process of evolution which invested most of the manufacturing industry over the last decades with the massive introduction of innovative technologies, such as CNC machines.

While the use of these tools has been introduced in architecture long time ago, they have not determined a productive paradigm shift. Indeed, buildings are in most of the cases assembled from sets of components which are gathered from catalogues provided by the industries.

Starting from the $90 \mathrm{~s}$ with the introduction of digital tools, the architectural designer's needs increased dramatically in terms of form personalization. Due to the fact that architecture cannot be built in one piece, and requires the prefabrication of components, it is automatically determined the crucial importance of the discretization of complex shapes characterizing the typical design workflow, in which architects conceive buildings just in terms of shape, and later on are facing problems related to construction feasibility.

Within this scenario, robotic fabrication has recently been discovered in the construction industry, after pioneering researches in the early 80s. Architects are especially interested in the robot ability to perform different tasks and in their low price compared to other machines. 


\section{Robotic Fabrication in Architecture. From Component Customization to Process Customization}

Typically the customization of building components is reached through design postadaptation, in order to fit industrial requirements. As a consequence of studies in the field of emerging technologies and bottom-up design the use of industrial robots has spread dynamically. Robots are multifunctional machines for mass-production, able to accomplish a wide range of works, but mainly used in the automotive industry where they usually do a single routine.

Robotic Fabrication in the construction field has by opposite the implicit advantage to be able of performing a multitude of tasks controlled from a common programming platform. With its employment, the logic of production and mass-customization can change radically for the construction field, shifting from specialized industrial media of production to versatile machine.

This has the potential to revolutionize the current understanding of masscustomization, moving the focus from geometrical post-optimization, to integration of robotic performance within the design process. The use of algorithms to control fabrication tools is natural extension of parametric modeling, helping the understanding of specific fabrication processes and in simulating the kinematics of the machine tool.

In the past few years, architectural faculties such as the ETH Zürich, TU Vienna, University of Stuttgart and the University of Michigan have acquired industrial robots and are actively researching the use of robots in architecture. Nowadays more than twenty architecture faculties in the world are experimenting in the use of industrial robots. However, this research is not limited to academia, with architectural offices like Snøhetta in Norway using robots in-house.

\section{$3 \quad$ Parametrically Controlled Robots}

The use of industrial robots would normally demand architects to migrate geometries from a CAD software to machines with a linear workflow, essentially converting geometries into working paths. This procedure is essentially transferring construction trajectories, and can be intended as limited in terms of interactions between design and production phases. This lack has recently been overcome by the development of new specific software tools, providing architects with an easier control from design environment to physical production and vice versa. The use of plug-ins such as KUKA PRC and Super KUKA for Grasshopper favors the integration into modeling environments of the dynamic of machines, simulating movements within the CAD space. Architects gained an increased capacity to control robots, and upcoming researches are moving in the direction of strengthening the interaction between design software and machines, in a full integration of the two systems. This paper proposes an overview on the different robotic fabrication procedures nowadays in development, proving how the flexibility of these machines enables architects to experiment a wide range of technical and aesthetic solutions. 


\section{Robotic Additive Fabrication}

Additive Fabrication represents the typical construction method employed in architecture and this is the main reason for the recent deep investigation which has been conducted for 3D-Printing at large scale, such as the Italian technology D-Shape and the American-based Contour Crafting. Additive processes can generally offer a wider potential over already established subtractive or formative digital fabrication processes, and in the last years various attempts in this direction have been made using robotic tools.

\subsection{Bricklaying Robotic Fabrication}

A popular example of additive fabrication is the Bricklaying Robot (2006) by Gramazio and Kohler which provides the architects with the possibility to generate brick walls designed parametrically. This system uses Kuka KR150 Robotic Arms to deposit bricks in simple and articulated wall compositions, basically replacing the traditional construction process based on manual work. In this case the robotic fabrication is improving fabrication speed, precision and expanding the formal freedom of the aesthetics of the construction. This technique has been applied for the façade of the Gantenbein Vineyard. In future projection, the authors plan to install the robots directly on building sites, combining the advantages of prefabrication, such as precision and high quality, with the advantages of short transport routes and just-intime production on the building site. Making use of computer methodologies in the design and fabrication process allows for manufacturing building elements with highly specific forms, which could not be built manually.

\section{Robotic Transformative Procedures}

\subsection{RoboFold Technology for Metal Sheets}

RoboFold is a patented method employed to form metal components with 6-axis industrial robots, especially referring to façade panels. The forming process is achieved by simultaneous work of robots folding sheet metal along curved crease lines, defined by project. The metal sheets are directly transformed by pressure applied on different directions, and no mold-tools are involved in the process. The system was invented by Gregory Epps in 2008, initially conceived for automotive industry and later transferred to construction field. The entire process is simulated in a CAD environment, this means that the design is performed with manufacturing constraints already taken into account since the design process.

Curve Folding is referring almost directly to the art of origami, paper engineering and industrial robot manufacturing. RoboFold uses Rhino3D software with the integration of Grasshopper parametric extension. Currently this system is developed through workshop and education system in various architectural faculties, and through the realization of façade mock-ups and furniture. 


\subsection{Cold Bending of Steel Rods}

An alternative transformative technique has been developed by Supermanouvre in collaboration with Matter Design, in order to produce an installation called "Clouds of Venice", exposed in Venice Biennale of Architecture 2012.

This installation is composed by a sequence of bended steel rods, in combination with a custom robotically-assisted $\mathrm{CNC}$ bender has been constructed and tested as an alternative to dedicated CNC forming equipment. This combination allowed for the serial creation of accurately defined bends, intervals and rotations. The robot provides the positioning while the bender applies the large forces required.

The operation is based on the proper disposition of three clamps: a collet-gripper mounted on the robot and two die clamps on the bender. One die clamp is used to provide pressure during actual bending, while the other two clamps alternate holding the stock in order to allow the robot to feed and rotate it into the appropriate position. The clamps must be capable of providing enough pressure and friction to counteract the torque resulting from the self-weight of the cantilevering rod.

This fabrication method can be easily implemented for the production of complex steel structures and for more standard applications such as steel reinforced concrete, where the precision and speed of robots can speed up operations which typically require longer time on site.

\section{Robot Subtractive Fabrication}

\subsection{Hot Wire Cutting Fabrication}

A hotwire cutter consists of a thin wire that is heated via an electric current to approximately 200 degrees Celsius and used to cut polystyrene or similar types of thermoplastic foam. The foam vaporizes just ahead of the wire and a minimal energy is required to cut through the stock. The robot-mounted bow-type hotwire has a series of specific constraints that must be embedded within the control software. The primary constraint is speed, while the primary simulation feedback is the positioning of the bow of the tool. The kerf width is directly proportional to the speed, so control over motion is extremely important. The input geometry is most often described by an upper and lower curve, generating a ruled loft between curves, finally generating ruled surfaces. By utilizing 7 axes of the robot simultaneously, large parts can be machined in one step.

A built example of this procedure is The Periscope Tower by Brandon Clifford and Wes Mcgee of Matter Design Studio, a temporary installation designed for the Young Architects Forum Atlanta. For the production a hotwire cutter was mounted onto the robot, creating a multi-axis CNC hotwire-cutter capable of processing 4 meters long EPS blocks. 
The designers sought a way to eliminate the excess waste beyond the efficiency of unit nesting, since the methods of fabrication produces no kerf waste and minimal waste which is $100 \%$ recyclable material. The fourteen meters height tower was assembled in only six hours.

\section{Conclusion and Outlook}

Despite the potential offered by digital fabrication with $\mathrm{CNC}$ machines, construction industry has rarely integrated innovative processes on a large scale, usually due to high costs products, machine low flexibility and time-related concerns. As consequence, complex shapes in architecture are often re-designed after project to fit the construction standard of particular machines. This paper shows an alternative to this typical workflow, focusing on how in the last years the development of robotic fabrication is consistently changing the production perspective towards a new professional paradigm where design software can be integrated with fabrication tools directly in the project.

Robots as universal and programmable machines offer a chance of highflexibility, and the analysis of the reported case studies highlights how the use of industrial robots move the attention from shape-oriented design, to material production system.

The use of robotic fabrication presents different advantages: flexible functionality, changing from a milling machine to a 3D-scanner just by switching its end-effectors; enlarged and geometrically customizable working space; affordable prices if compared with multi-axis Computer Numeric Control (CNC) machines.

This system has yet to compete with mass production and its associated economies of scale in fabricating widely distributed products, but they have already shown the potential to empower customized solutions. These devices can be tailored to local or personal needs in ways that are not practical or economical using mass production lines.

The actual use of robotics under the experiments of several pioneers highlights promising potential and results, but still without a clear vision on how these new tools can be implemented on industrial scale. Within an industrial perspective, the implicit risk of these experiments is to create a new design/fabrication niche, unable to determine an effective impact on the production systems.

In this sense the contemporary use of robotics is limited to the idea of advanced crafting, and the integration on wider scale seems currently hard to be achieved. The analyzed case studies open the idea of "robotic crafting", which could be further adapted to be performed within the context of the building site, more than as a media of industrial prefabrication. Within this scenario it appears fundamental the implementation of more accessible software, in order to be used by less specialized operators. 


\section{References}

1. Aigner, A., Brell-Cokcan, S.: Surface Structures and Robot Milling. The Impact of Curvilinear Structured Architectural Scale Models on Architectural Design and Production. In: Ingrid, P. (ed.) Innovative Design and Construction Technologies. Building complex shapes and beyond, pp. 433-445. Maggioli Editore, Milano (2009)

2. Bonwetsch, T., Gramazio, F., Kohler, M.: The informed wall, applying additive digital fabrication techniques on architecture. In: Proceedings of the 25th Annual Conference of the Association for Computer-Aided Design in Architecture, Louisville, pp. 489-495 (2006)

3. Johannes, B., Sigrid, B.-C.: Digital and Physical Computing for Industrial Robots in Architecture. Interfacing Arduino with industrial robots. In: Beyond Codes and Pixels: Proceedings of the 17th International Conference on Computer-Aided Architectural Design Research in Asia, Hong Kong, pp. 317-326 (2012)

4. Sigrid, B.-C., Johannes, B.: Computer Numeric Controlled Manufacturing for Freeform Surfaces in Architecture. In: Kuhlmann, D., Brell-Cokcan, S., Schinegger, K. (eds.) Emotion in Architecture, pp. 20-25. Institut fur Architekturwissenschaften, Wien (2011)

5. Sigrid, B.-C., Johannes, B.: Parametric Robot Control. Intergrated CAD/CAM for architectural design. In: ACADIA 2011: Integration through Computation. Proceedings of the 31st Annual Conference of the Association for Computer Aided Design in Architecture, Banff, pp. 242-251 (2011)

6. Sigrid, B.-C., Johannes, B.: A New Parametric Design Tool for Robot Miling. In: ACADIA 2010: LIFE in:formation, On Responsive Information and Variations in Architecture. Proceedings of the 30th Annual Conference of the Association for Computer Aided Design in Architecture, New York, pp. 357-363 (2010)

7. Sigrid, B.-C., Martin, R., Heinz, S., Johannes, B.: Digital Design to Digital Production. In: 27th eCAADe Conference Proceedings, Session 2009: Modes of Production, Istanbul, pp. 323-329 (2009)

8. Kaczynski Maciej, P., Wes, M., Dave, P.: Robotically Fabricated Thin-shell Vaulting. A method for the integration of multi-axis fabrication processes with algorithmic formfinding techniques. In: ACADIA 2011: Integration through Computation. Proceedings of the 31st Annual Conference of the Association for Computer Aided Design in Architecture, Banff, pp. 114-121 (2011)

9. Knaack, U., Bilow, M., Klein, T.: Rapids. Imagine 04. 010 Publishers, Rotterdam (2010)

10. Kolarevic, B. (ed.): Architecture in the Digital Age. Design and Manufacturing. Taylor \& Francis, New York (2005)

11. David, K.O., Karola, D., Steffen, R., Tobias, S., Achim, M.: Performative Architectural Morphology. Robotically manufactured biomimetic finger-joined plate structures. In: 29th eCAADe Conference Proceedings. New Design Concepts and Strategies, Ljubljana, pp. 573-580 (2011)

12. Justin, L., Rachel, V., Yair, K.: Automated Folding of Sheet Metal Components with a Six-axis Industrial Robot. In: ACADIA 2011: Integration through Computation. Proceedings of the 31st Annual Conference of the Association for Computer Aided Design in Architecture, Banff, pp. 144-151 (2011) 
13. Parke, M., Diana, T.: Robotic Rod-bending. Digital drawing in physical space. In: ACADIA 2011: Integration through Computation. Proceedings of the 31st Annual Conference of the Association for Computer Aided Design in Architecture, Banff, pp. 132-137 (2011)

14. Andrew, P.: A Five-axis Robotic Motion Controller for the Designers. In: ACADIA 2011: Integration through Computation. Proceedings of the 31st Annual Conference of the Association for Computer Aided Design in Architecture, Banff, pp. 162-169 (2011)

15. Dave, P., Wes, M.: Formation Embedded Design. A methodology for the integration of fabrication constraints into architectural design. In: ACADIA 2011: Integration through Computation. Proceedings of the 31st Annual Conference of the Association for Computer Aided Design in Architecture, Banff, pp. 122-131 (2011) 